\title{
O ÍNDICE DE RISCO NUTRICIONAL (NUTRITIONAL RISK INDEX) É PREDITOR DE COMPLICAÇÃO PÓS-OPERATÓRIA EM OPERAÇÕES DO APARELHO DIGESTIVO OU PAREDE ABDOMINAL?
}

\author{
Nutritional risk index is predictor of postoperative complications in operations of digestive system or abdominal wall? \\ Rubia Daniela THIEME ${ }^{1}$, Gislaine CUTCHMA ${ }^{1}$, \\ Maria Eliana Madalozzo CHIEFERDECKER ${ }^{1}$, Antônio Carlos Ligocki CAMPOS ${ }^{1,2}$
}

Trabalho realizado no ${ }^{1}$ Departamento de Nutrição e '2Departamento de Cirurgia, Setor de Ciências da Saúde, Universidade Federal do Paraná, Curitiba, PR, Brasil.

DESCRITORES - Desnutrição. Avaliação nutricional. Sistema digestório. Parede abdominal. Complicações pós-operatórias.
RESUMO - Racional : Pode-se considerar a desnutrição como doença mais comum no ambiente hospitalar, devido à sua alta prevalência. Objetivo: Verificar os indicadores de estado nutricional que melhor se correlacionam com as complicações pós-operatórias e o tempo de permanência hospitalar de pacientes submetidos à operações do aparelho digestivo ou parede abdominal de médio e grande porte. Método: Estudo retrospectivo, com análise de 215 fichas de avaliação nutricional de pacientes cirúrgicos que foram submetidos à avaliação nutricional por meio de dados antropométricos (peso, altura, IMC, circunferência do braço, prega cutânea triciptal, circunferência muscular do braço), avaliação subjetiva global e dados bioquímicos (contagem total de linfócitos e albumina sérica). Em adição, posteriormente foi calculado o índice de risco nutricional. Resultados: Foram incluídos 125 indivíduos. Diagnóstico de desnutrição de acordo com circunferência muscular do braço, índice de risco nutricional e avaliação subjetiva global foi de $46 \%$, $88 \%$ e $66 \%$, respectivamente. A classificação como gravemente desnutridos foi de $17,6 \%$ dos pacientes de acordo com a avaliação subjetiva global, enquanto que com o índice de risco nutricional foi de $42 \%$. Os pacientes com câncer são mais desnutridos (5,42 unidades do índice de risco nutricional a menos). Houve correlação significativa entre ocorrência de complicações pós-operatórias não infecciosas quando analisado o índice de risco nutricional, considerando que seus menores complicaram mais $(p=0,0016)$. O mesmo resultado foi obtido para albumina sérica $(p=0,0015)$. Os pacientes sem complicações permaneceram, em média, 14,24 dias internados a menos do que os pacientes com complicações não infecciosas $(p<0,05)$. Conclusão: $O$ índice de risco nutricional e a albumina sérica são os parâmetros com melhor capacidade em predizer ocorrência de complicações pós-operatórias não infecciosas.

\section{Correspondência: \\ Rubia Daniela Thieme \\ E-mail: rubiathieme@gmail.com \\ Fonte de financiamento: não há \\ Conflito de interesses: não há}

Recebido para publicação: 02/05/2013

Aceito para publicação: 08/08/2013

HEADINGS - Malnutrition. Nutrition assessment. Digestive system. Abdominal wall. Postoperative complications.
ABSTRACT - Background: Malnutrition can be considered the most common disease in hospitals due to its high prevalence. Aim: To investigate the methods of evaluation of the nutritional status that better correlate with postoperative complications and the length of hospital stay in patients submitted to gastrointestinal or abdominal wall surgeries. Methods: This is a retrospective evaluation of 215 nutritional assessment records. All were submitted to traditional anthropometry (weight, height, BMI, arm circumference, triceps skinfold thickness and mid-arm muscle circumference), subjective global assessment, serum albumin and lymphocyte count. Nutritional risk index was also calculated. Results: A total of 125 patients were included. Malnutrition was diagnosed by mid-arm muscle circumference, nutritional risk index and subjective global assessment in $46 \%, 88 \%$ and $66 \%$, respectively. Severe malnutrition was found in $17,6 \%$ if considered subjective global assessment and in $42 \%$ by the nutritional risk index. Oncologic patients had a worst nutritional status according to this index (5,42 less units). There was a negative correlation between occurrence the noninfectious postoperative complications with the nutritional risk index $(p=0,0016)$. Similarly, lower serum albumin levels were associated with higher non infectious complications ( $p=0,0015)$. The length of hospital stay was, in average, 14,24 days less in patients without complications as compared with non infectious postoperative complications $(p<0,05)$. Conclusion: Nutritional risk index and serum albumin are the parameters with the best capacity to predict the occurrence of non infectious postoperative complications and the length of hospital stay was higher to this patients. 
INTRODUÇÃO

$\mathrm{P}$ ode-se considerar a desnutrição como doença mais comum no ambiente hospitalar, devido à sua alta prevalência. Estudos clássicos como o ELAN e o IBRANUTRI, na América Latina e no Brasil, respectivamente, apontam que cerca de $50 \%$ dos indivíduos hospitalizados apresentam algum grau de desnutrição. A desnutrição grave manifesta-se em aproximadamente 12\% dos pacientes. Nas doenças gastrointestinais, ela acomete $61,5 \% 11,18,28,29$. Os pacientes submetidos a operações do aparelho digestivo ou parede abdominal de médio ou grande porte, frequentemente possuem estado nutricional comprometido ${ }^{16,26}$.

Outro fator relevante é o trauma cirúrgico, que aumenta o catabolismo e a demanda metabólica. O risco de desnutrição também aumenta com a idade superior a 60 anos, na presença de infecção e inflamação. Instalada a desnutrição, ela associa-se ao aumento da morbidade, risco de complicações, reintervenção cirúrgica mais frequente, maior tempo e custo de hospitalização e à mortalidade $\mathrm{e}^{4,9,28,29}$.

Frequentemente, o cirurgião e o nutricionista questionam se o paciente teve perda ponderal recente por já saber que esse indivíduo pode estar desnutrido e, portanto, mais vulnerável à complicação 20,27,29. A identificação precoce da desnutrição permite o planejamento da terapia nutricional apropriada, que é capaz de prevenir, interromper ou reverter o estado nutricional comprometido e evitar complicações decorrentes da operação $0^{6,16,29}$.

A avaliação nutricional deve ser capaz de predizer o desfecho clínico ou prognóstico do paciente e, ao mesmo tempo, apresentar baixo custo e rápida realização. Os métodos de avaliação do estado nutricional incluem dados antropométricos, dietéticos, bioquímicos, imunológicos, história clínica, avaliação subjetiva e exame físico. Entre os parâmetros antropométricos clássicos estão: peso habitual, peso atual, percentual de perda de peso recente, IMC, métodos de detecção de massa muscular a tecido adiposo subcutâneo, tais como as circunferências corporais (circunferência do braço e circunferência muscular do braço) e pregas cutâneas (prega cutânea triciptal), competência imune (contagem total de linfócitos), proteínas séricas, sobretudo albumina ${ }^{28}$. Para verificar os índices prognósticos são utilizados a avaliação subjetiva global (ASG) e o índice de risco nutricional (IRN) $)^{8,12}$.

Estudos têm demonstrado que avaliar isoladamente estes indicadores nutricionais pode gerar falhas, mesmo aqueles considerados padrão-ouro, como a $\mathrm{ASG}^{12}$. Assim, a análise do conjunto destes parâmetros até hoje é a melhor maneira de estabelecer o diagnóstico nutricional ${ }^{6}$. As pesquisas nesta área buscam estabelecer um índice isolado como padrão-ouro para identificação de risco nutricional e desfecho clínico 25 .

Os objetivos deste estudo foram verificar os indicadores de estado nutricional que melhor se correlacionam com as complicações pós-operatórias e o tempo de permanência hospitalar de pacientes submetidos à operações do aparelho digestivo ou parede abdominal de médio e grande porte.

\section{MÉTODOS}

O estudo foi aprovado pelo Comitê de Ética em Pesquisa em Seres Humanos do Hospital de Clínicas da Universidade Federal do Paraná - HCUFPR sob n. CAAE: 04125312.8.0000.0096. Caracteriza-se como pesquisa retrospectiva com análise inicial de 215 fichas de avaliação nutricional de indivíduos admitidos nas unidades de Cirurgia do Aparelho Digestivo e de Cirurgia Geral do HCUFPR, entre janeiro de 2010 e outubro de 2011, para realização de procedimento cirúrgico do aparelho digestivo ou de parede abdominal de médio ou grande porte. Para as análises e valores de referências os indivíduos com idade superior a 60 anos foram considerados idosos. Foram excluídos os indivíduos com idade abaixo de 18 anos, os que não foram submetidos à operação, os submetidos a procedimento de pequeno porte, a operações não abdominais ou digestivas e aqueles que possuíam avaliação nutricional incompleta. Consideraram-se complicações pós-operatórias anormalidades que acorreram no período após a operação, podendo ser não infecciosas ou infecciosas. As complicações não infecciosas foram aquelas em que houve exacerbação de doenças prévias ou insuficiências orgânicas. Como infecciosas consideraram-se sepse, pneumonia, infecção da ferida operatória e fístula. Estas foram consideradas como contaminadas e com probabilidade grande de infecção, por isso, foram consideradas como complicação infecciosa ${ }^{20}$. Quando havia mais de uma complicação, foi considerada a mais grave ou a infecciosa para a análise.

Do Sistema de Informação Hospitalar informatizado foram retirados os dados de exames bioquímicos, diagnóstico médico, operação realizada, complicações pós-operatórias, necessidade de reintervenção cirúrgica, dia de admissão hospitalar, dia de alta hospitalar e óbito.

A avaliação nutricional foi realizada por nutricionistas da Unidade de Nutrição e Dietética ou residentes em nutrição do Programa de Residência Multiprofissional conforme protocolo referendado préestabelecido, preferencialmente no pré-operatório; porém, para operações de emergência a avaliação nutricional foi realizada no pós-operatório. Destas fichas foram coletados dados de identificação do paciente, como nome, número de registro hospitalar, sexo e idade. Foram levantados para este estudo: ASG, dados antropométricos, como, peso, altura, 
IMC, circunferência do braço, prega cutânea triciptal, circunferência muscular do braço, e dados bioquímicos, como contagem total de linfócitos e albumina. Os dados de avaliação nutricional utilizados foram os contidos nas fichas arquivadas pela unidade de nutrição clínica. Os métodos dessa avaliação foram adaptados conforme estado geral do paciente no momento da avaliação. Assim, para alguns pacientes foi necessário utilização de equações validadas para estimar a estatura quando os pacientes não eram capazes de deambular ${ }^{10}$.

O IRN foi estabelecido durante tabulação dos dados contidos nas fichas de avaliação nutricional.

\section{Avaliação Subjetiva Global}

A ASG foi adaptada de Detsky et al. ${ }^{12}$, em 1987. Proporciona integração entre de dados da história clínica recente e dados físicos (histórico de perda de peso, alteração na ingestão alimentar, sintomas gastrointestinais, capacidade funcional ou nível de energia do paciente, análise de perda de tecido adiposo subcutâneo e tecido muscular e presença de edema ou ascite). Com base nesta avaliação, os pacientes foram classificados em nutridos (ASG A), risco de desnutrir ou desnutrição moderada (ASG B) ou desnutrição grave (ASG C) ${ }^{12}$.

\section{Medidas antropométricas}

De acordo com o protocolo pré-estabelecido, o peso corporal atual foi aferido com balança digital e a estatura foi real ou estimada por chanfradura ou altura do joelho ${ }^{10,13}$, com o paciente sem sapatos e roupas leves. Após, o IMC $\left(\mathrm{Kg} / \mathrm{m}^{2}\right)$ foi calculado com base na classificação do Ministério da Saúde e Organização Mundial da Saúde, sendo diferenciado para os indivíduos com idade superior aos 60 anos.

$O$ percentual de perda de peso foi calculado a partir da equação: (Peso habitual $(\mathrm{Kg})$ - Peso atual $(\mathrm{Kg}) /$ Peso habitual $(\mathrm{Kg}))$ x 100 e considerou-se perda de peso grave quando superior a $10 \%$ em seis meses ${ }^{2}$. A circunferência do braço foi aferida no ponto médio entre o acrômio e o olécrano do membro superior direito com a musculatura relaxada utilizando-se fita inelástica $(0,1 \mathrm{~cm})$. No mesmo ponto, foi aferida a prega cutânea triciptal utilizando adipômetro científico. Após a obtenção da circunferência do braço e prega cutânea triciptal, foi calculada a circunferência muscular do braço através da equação: circunferência muscular do braço $(\mathrm{cm})=$ circunferência do braço $(\mathrm{cm})-(0,314 \times$ prega cutânea triciptal $(\mathrm{mm})$ ). Para as pregas e circunferências foram realizadas as adequações conforme sexo e idade. Foi considerada adequação quando o valor encontrado foi superior a $90 \%$ da referência. Para valores menores, considerou-se desnutrição leve entre 80 e 90\%, moderada entre 70 e $80 \%$ e grave inferior a $70 \%{ }^{3}$.

\section{Exames bioquímicos}

As amostras sanguíneas foram coletadas pelo serviço de coleta do hospital. Foram dosados albumina e contagem total de linfócitos. Para albumina sérica, consideraram-se os seguintes valores: acima de $3,5 \mathrm{~g} / \mathrm{dl}$ normal, entre 3 e 3,5 g/dl depleção leve, entre 2,4 e 2,9 $\mathrm{g} / \mathrm{dl}$ depleção moderada e abaixo de $2,4 \mathrm{~g} / \mathrm{dl}$, depleção grave. Os parâmetros para classificação considerando reservas imunológicas a partir da contagem total de linfócitos foram: acima de $2000 \mathrm{~mm}^{3}$ sem depleção; entre 1200 e $2000 \mathrm{~mm}^{3}$, depleção leve; entre 800 e $1199 \mathrm{~mm}^{3}$, depleção moderada e inferior à $800 \mathrm{~mm}^{3}$, depleção grave $\mathrm{g}^{2,5}$.

\section{Índice de risco nutricional}

Como novo parâmetro de avaliação do estado nutricional, foi aplicada equação para o cálculo do IRN, que utiliza os valores de albumina sérica e percentual de perda de peso: $\operatorname{IRN}=[1.519 \times$ albumina sérica $(\mathrm{g} / \mathrm{l})]+$ $0.417 \times($ peso atual/peso habitual $\times 100)$. Foi realizada categorização de acordo com o IRN. Resultado acima de 100 indicava nutrição satisfatória; entre 97,5 e 100, desnutrição leve; de 83,5 a 97,5 desnutrição moderada; e abaixo de 83,5 , desnutrição grave ${ }^{8,25}$.

\section{Análise estatística}

As médias estão apresentadas juntamente com o desvio-padrão. As análises estatísticas foram realizadas por meio o software R 2.14.0. A comparação entre duas variáveis categóricas deu-se por teste do QuiQuadrado. Quando a variável dependente e a variável explicativa fossem das ordens ordinal e categórica, respectivamente, foi aplicado o teste da ANOVA, sendo que para identificação de diferenças entre grupos utilizou-se teste de Turkey, de Kruskal-Wallis e de Comparações Múltiplas, e como alternativa para ANOVA utilizou-se Teste $U$ de Mann-Whitney. Para os casos em que ambas as variáveis fossem ordinárias, a associação foi verificada diretamente, por meio do teste de Correlação de Pearson e de Spearman. Foram consideradas diferenças estatisticamente significativas se $p \leq 0,05$.

\section{RESULTADOS}

Dos 215 indivíduos, foram incluídos no estudo 125 pacientes que se enquadraram nos critérios estabelecidos (Tabela 1).

A média de idade foi de $58,5 \pm 14,9$ anos, sendo que $49 \%$ dos indivíduos eram idosos. Cerca de metade $(49,6 \%)$ do total víduos tinha diagnóstico de doença maligna e, entre os maiores de 60 anos, o número aumentou para 59,7\%. Dentre as doenças não oncológicas, foi observada prevalência delas no esôfago (megaesôfago), estômago (úlcera gástrica perfurada), vias biliares (colelitíase e colangite) e intestino (hérnias e apendicites complicadas, exacerbação de doença de Crohn). As fístulas foram causa de admissão hospitalar para 4\% dos pacientes. Os tipos de câncer mais comuns foram os da região esofagogástrica e intestinal em ambos os sexos. 
TABELA 1 - Características da amostra, separadas por gênero e total

\begin{tabular}{|c|c|c|c|}
\hline Variável & $\begin{array}{l}\text { Masculino } \\
n=77\end{array}$ & $\begin{array}{l}\text { Feminino } \\
n=48\end{array}$ & $\begin{array}{c}\text { Total } \\
\mathrm{n}=125\end{array}$ \\
\hline Idade & $58,7 \pm 15$ & $58,1 \pm 14,9$ & $58,5 \pm 14,9$ \\
\hline $\begin{array}{c}\text { Doenças não } \\
\text { oncológicas } \\
\text { Gastroesofágicas } \\
\text { Hepáticas } \\
\text { Vias biliares } \\
\text { Pancreáticas } \\
\text { Intestinais } \\
\text { Hérnias complicadas } \\
\text { Fístulas enterocutâneas } \\
\text { Fístula biliar }\end{array}$ & $\begin{array}{c}45,5 \% \\
9,1 \% \\
3,9 \% \\
14,3 \% \\
5,2 \% \\
11,7 \% \\
3,9 \% \\
3,9 \% \\
1,3 \%\end{array}$ & $\begin{array}{c}58,3 \% \\
14,6 \% \\
2,1 \% \\
10,4 \% \\
2,1 \% \\
14,6 \% \\
6,2 \% \\
2,1 \% \\
0\end{array}$ & $\begin{array}{c}50,4 \% \\
11,2 \% \\
4,0 \% \\
12,8 \% \\
4,0 \% \\
14,4 \% \\
4,8 \% \\
3,2 \% \\
0,8 \%\end{array}$ \\
\hline $\begin{array}{l}\text { Doença oncológica } \\
\text { Gastroesofágicas } \\
\text { Hepáticas } \\
\text { Vias biliares } \\
\text { Pancreáticas } \\
\text { Intestinais }\end{array}$ & $\begin{array}{c}54,5 \% \\
20,8 \% \\
5,2 \% \\
1,3 \% \\
1,3 \% \\
28,6 \%\end{array}$ & $\begin{array}{c}41,7 \% \\
14,6 \% \\
0 \\
0 \\
4,2 \% \\
20,8 \%\end{array}$ & $\begin{array}{c}49,6 \% \\
18,4 \% \\
3,0 \% \\
0,8 \% \\
2,0 \% \\
25,6 \%\end{array}$ \\
\hline $\begin{array}{l}\text { Complicação pós- } \\
\text { operatórias } \\
\text { Infecciosa } \\
\text { Não infecciosa } \\
\text { Re-intervenção } \\
\text { cirúrgica }\end{array}$ & $\begin{array}{l}55,9 \% \\
26,0 \% \\
29,9 \% \\
10,4 \%\end{array}$ & $\begin{array}{c}41,7 \% \\
27,1 \% \\
14,6 \% \\
6,3 \%\end{array}$ & $\begin{array}{c}50,4 \% \\
26,4 \% \\
24,0 \% \\
8,8 \%\end{array}$ \\
\hline $\begin{array}{l}\text { HAS } \\
\text { DM }\end{array}$ & $\begin{array}{l}37,7 \% \\
221 \%\end{array}$ & $\begin{array}{l}35,4 \% \\
146 \%\end{array}$ & $\begin{array}{l}36,8 \% \\
19,2 \%\end{array}$ \\
\hline $\mathrm{GJ}(\mathrm{mg} / \mathrm{dL})( \pm \mathrm{DP})$ & $107 \pm 37,6$ & $100,5 \pm 28,5$ & $104,5 \pm 34,4$ \\
\hline \%PP $( \pm D P)$ & $9,1 \pm 9,1$ & $10,8 \pm 12,5$ & $9,7 \pm 10,5$ \\
\hline CTL $(\mathrm{mm} 3)( \pm \mathrm{DP})$ & $1535 \pm 989,8$ & $1824,3 \pm 1192,5$ & $1642,6 \pm 1076,8$ \\
\hline Albumina sérica (g/dL) ( $\pm \mathrm{DP})$ & $3,06 \pm 0,73$ & $3,22 \pm 0,66$ & $3,12 \pm 0,70$ \\
\hline IRN (unidades) ( \pm DP) & $84 \pm 13$ & $86,1 \pm 13,3$ & $84,1 \pm 13,1$ \\
\hline Hospitalização (dias) ( \pm DP) & $21,9 \pm 25,4$ & $16,7 \pm 21,1$ & $19,9 \pm 23,9$ \\
\hline
\end{tabular}

Após a operação $50,4 \%$ tiveram complicações relacionadas tanto com o ato cirúrgico quanto com a exacerbação de doenças crônicas. A metade das complicações foi infecciosa, acometendo $26,4 \%$ dos pacientes, enquanto em $24 \%$ eram não infecciosas, as quais foram mais prevalentes nos homens comparado às mulheres (29,9\%vs14,6\%). Das complicações pósoperatórias, encontraram-se principalmente fístulas em 18 pacientes, complicação pulmonar não infecciosa em 11; menor número de pacientes apresentaram eventração, deiscência, infecção de ferida operatória ou coleção, sepse de diferentes focos, disfunção renal, pneumonia e insuficiência cardíaca. Dos pacientes que tiveram complicação, 17,5\% necessitaram de nova intervenção cirúrgica e 14,4\% morreram durante a hospitalização.

Quanto ao estado nutricional, considerando os parâmetros/indicadores isoladamente, verificaram-se diferentes resultados quanto à presença de depleção nutricional, incluindo leve, moderada e grave, como pode ser observado na Figura 1 . O diagnóstico de desnutrição foi de apenas $8 \%$ segundo o IMC, de $66 \%$ de acordo com a ASG e de até $88 \%$ para o IRN. Os valores médios encontrados para albumina sérica, contagem total de linfócitos e IRN indicaram que os pacientes apresentavam depleção nutricional de acordo com esses parâmetros (Tabela 1).

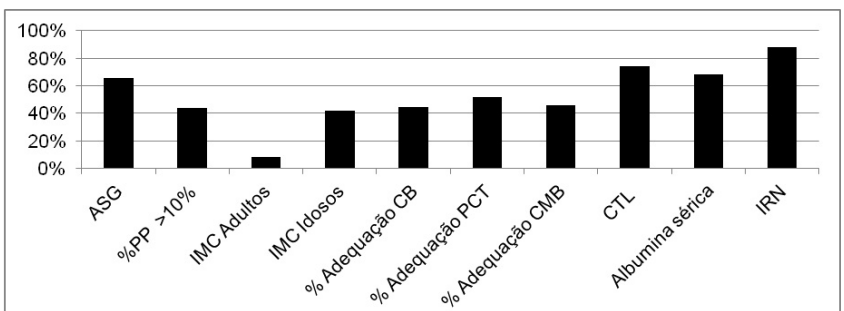

Legenda - ASG: avaliação subjetiva global; \%PP> 10\%: percentual de perda de peso superior a $10 \%$ em seis meses; IMC Adultos: índice de massa corporal $\left(\mathrm{Kg} / \mathrm{m}^{2}\right)$ para adultos; IMC Idosos: índice de massa corporal $\left(\mathrm{Kg} / \mathrm{m}^{2}\right)$ para idosos; \% Adequação CB: percentual de adequação da circunferência do braço; \% Adequação PCT: percentual de adequação da prega putânea triciptal; \% Adequação CMB: percentual de adequação da circunferência muscular do braço;CTL: contagem total de linfócitos; IRN: índice de risco nutricional

FIGURA 1 - Diagnóstico de desnutrição de acordo com diferentes parâmetros de avaliação nutricional em percentual de indivíduos

A classificação como gravemente desnutridos, de acordo com a ASG C, ocorreu em $17,6 \%$ dos pacientes, enquanto que, de acordo com o IRN, em 42\%. Parâmetro exclusivamente antropométrico, a adequação da circunferência muscular do braço demonstrou $46,4 \%$ de desnutrição, com $6,4 \%$ de desnutridos graves, enquanto que a prega cutânea triciptal resultou em $52,2 \%$ dos indivíduos com algum grau de desnutrição, sendo que 31,2\% apresentaram desnutrição grave. Os parâmetros bioquímicos demonstraram que a competência imunológica esteve comprometida em $74,4 \%$ dos pacientes e para $25,6 \%$ deles o comprometimento era considerado grave. Quanto às proteínas séricas, os níveis de albumina indicando comprometimento do estado nutricional foram encontrados em $68 \%$, com $15,2 \%$ de comprometimento grave (Tabela 2 ).

TABELA 2 - Desnutrição segundo indicador de estado nutricional e nível de gravidade da desnutrição

\begin{tabular}{|c|c|c|c|c|c|c|c|}
\hline 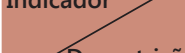 & ASG & $\% \mathrm{CB}$ & $\%$ PCT & $\% \mathrm{CMB}$ & CTL & Albumina & IRN \\
\hline Grave & $17,6 \%$ & $8 \%$ & $31,2 \%$ & $6,4 \%$ & $15,2 \%$ & $15 \%$ & $42 \%$ \\
\hline Moderada & $48 \%$ & $12,8 \%$ & $11 \%$ & $10 \%$ & $23,2 \%$ & $22 \%$ & $39 \%$ \\
\hline Leve & * & $24 \%$ & $10 \%$ & $30 \%$ & $36 \%$ & $31 \%$ & $7 \%$ \\
\hline Não desnutrido & $34,4 \%$ & $55,2 \%$ & $48 \%$ & $54 \%$ & $25,6 \%$ & $32 \%$ & $12 \%$ \\
\hline
\end{tabular}

Legenda - ASG: avaliação subjetiva global; \%CB: percentual de adequação da circunferência do braço; \%PCT: percentual de adequação da prega cutânea triciptal; \%CMB: percentual de adequação da circunferência muscular do braço; CTL: contagem total de linfócitos; IRN: índice de risco nutricional; *: sem classificação de desnutrição leve para este indicador

A classificação do estado nutricional de acordo com a ASG não associou-se com a presença de complicação pós-operatória $(p=0,6471)$ nem com reintervenção cirúrgica $(p=0,4361)$, presença de doença oncológica $(p=0,3854)$ e ocorrência de óbito durante a internação $(p=0,0719)$.

Os dados antropométricos mostraram que segundo o IMC, $8 \%$ dos adultos eram desnutridos e $42 \%$ dos idosos apresentavam baixo peso. Entretanto, 
não existem diferenças estatisticamente significativas dos valores de IMC entre os grupos de pacientes, tanto entre os adultos $(p=0,7324)$ como entre os idosos $(p=0,4422)$, com ou sem complicações cirúrgicas. Quanto à reserva muscular e adiposa, a composição corporal avaliada por meio de prega cutânea triciptal e circunferência muscular do braço não demonstrou diferença significativa entre o grupo que apresentou complicação pós-operatória e o que não apresentou (para circunferência muscular do braço $p=0,8471$ e para prega cutânea triciptal $p=0,2205$ ). A competência imunológica verificada por meio da contagem total de linfócitos, também não se correlacionou com as complicações pós-operatórias, sendo nula inclusive para as infecciosas $(p=0,9105)$.

Pôde-se observar, por outro lado, que houve correlação estatisticamente significativa entre os valores encontrados para o IRN e as complicações pósoperatórias não infecciosas comparados com aqueles que não complicaram, sendo que menores resultados de IRN complicaram mais $(p=0,0016)$. Essa diferença foi de 9,52 unidades a menos, em média, comparado aos que não apresentaram complicações (Tabela 3). Porém, os resultados não foram significativos para as complicações infecciosas, entre os tipos de complicação e necessidade de re-operação $(p=0,2429)$ ou mortalidade $(p=0,1277)$.

TABELA 3 - Comparação das médias de IRN e albumina sérica e desfechos pós-operatórios

\begin{tabular}{|l|c|c|}
\hline Desfecho pós-operatório & $\begin{array}{c}\text { Média de IRN } \\
\text { (unidades) }\end{array}$ & $\begin{array}{c}\text { Média de albumina } \\
\text { sérica }(\mathrm{g} / \mathrm{dl})\end{array}$ \\
\hline Sem complicações & $88,43^{\mathrm{a}}$ & $3,30 \mathrm{~b}$ \\
\hline Complicação infecciosa & 84,38 & 3,11 \\
\hline Complicação não infecciosa & $78,91^{\mathrm{a}}$ & $2,77 \mathrm{~b}$ \\
\hline
\end{tabular}

a Diferença estatisticamente significativa: $p=0,0016$

${ }^{b}$ Diferença estatisticamente significativa: $p=0,0015$

Houve também correlação significativa entre baixos valores de albumina e complicações pósoperatórias não infecciosas, comparados àqueles que não apresentaram complicações ( $p=0,0015)$ (Tabela 3).

Os pacientes com doença oncológica possuem IRN 5,42 unidades, em média, menor do que os com doenças não malignas, demonstrando que pacientes com câncer são mais desnutridos. Houve diferença estatisticamente significativa entre oncológicos com complicações não infecciosas e não oncológicos e sem complicações, que apresentaram em média 8,46 unidades de IRN a menos $(p=0,0053)$ (Tabela 4). Esse resultado é similar ao resultado encontrado quando não se faz a distinção entre doença oncológica ou não oncológica.

O tempo médio de permanência hospitalar foi de 19,9 $\pm 23,9$ dias (Tabela 1). Depois de aplicada análise estatística multivariada, observou-se que os pacientes sem complicações permaneceram, em média, 7,56 dias e 14,24 dias internados a menos do que os com complicações infecciosas e não infecciosas, respectivamente, com $p<0,05$. Entretanto, não há diferenças significativas no tempo de internação entre os pacientes com complicações infecciosas e não infecciosas (Tabela 5).

TABELA 4 - Médias de IRN por doença oncológica e não oncológica para presença e tipo de complicações pós-operatórias

\begin{tabular}{|c|c|c|c|}
\hline \multirow[b]{2}{*}{$\begin{array}{l}\text { Tipo de } \\
\text { doença }\end{array}$} & \multicolumn{3}{|c|}{ Médias de IRN nos desfechos pós-operatórios } \\
\hline & $\begin{array}{c}\text { Sem } \\
\text { complicação }\end{array}$ & $\begin{array}{c}\text { Complicação } \\
\text { infecciosa }\end{array}$ & $\begin{array}{l}\text { Complicação } \\
\text { não infecciosa }\end{array}$ \\
\hline Oncológica & 85,84 & 81,29 & $78,99^{*}$ \\
\hline $\begin{array}{c}\text { Não } \\
\text { oncológica }\end{array}$ & $89,96^{*}$ & 90,57 & 78,82 \\
\hline
\end{tabular}

*Diferença estatisticamente significativa: $p=0,0053$

TABELA 5 - Média de tempo de permanência hospitalar por presença e tipo de complicação pós-operatória (infecciosa ou não infecciosa)

\begin{tabular}{|cc|}
\hline Desfechos pós-operatórios & $\begin{array}{c}\text { Tempo de permanência } \\
\text { hospitalar (dias) }\end{array}$ \\
\hline Sem complicação & $14,53^{*}$ \\
\hline Complicação infecciosa & 22,09 \\
\hline Complicação não infecciosa & $28,77^{\star}$ \\
\hline
\end{tabular}

*Diferença estatisticamente significativa: $p>0,05$

\section{DISCUSSÃO}

As dificuldades em se desenvolver pesquisas que demonstrem resultados satisfatórios e confiáveis relacionando estado nutricional e operações de médio e grande porte são decorrentes principalmente das diversas variáveis que podem influenciar nos resultados, como idade e gravidade da doença, e da variabilidade de indicadores nutricionais que podem ser utilizados.

A população do presente estudo foi homogênea no quesito idade entre os gêneros, e metade da população era composta por idosos, assim como quanto a doenças oncológicas ou não oncológicas. Por ter sido realizada em hospital de alta complexidade, a maioria dos casos foi considerada grave. As maiores discrepâncias foram justamente encontradas naquilo que é um dos principais focos desse estudo: a variabilidade dos métodos de avaliação do estado nutricional. A dificuldade de concordância entre os indicadores resultou em diferentes correlações com os desfechos cirúrgicos.

Os resultados para desnutrição do presente estudo, quando se considera a gravidade da desnutrição de acordo com ASG (classificados como C) mostrou valor superior $(17,6 \%)$ ao encontrado por Pablo e et al. ${ }^{23}$, de 8,3\% de desnutrição. Diferente do estudo realizado por Ryu e $\mathrm{Kim}^{25}$ em que observaram a presença de desnutrição em $31 \%$ dos indivíduos com câncer gástricos submetidos a tratamento cirúrgico curativo e do IBRANUTRI, estudo em que os resultados apontaram 39\% de desnutridos entre os pacientes cirúrgicos no Brasil $^{25,28}$. 
Ao comparar o percentual de desnutridos graves segundo ASG e IRN observa-se que o percentual foi alto para ambos, $17,6 \%$ e $42 \%$ respectivamente; porém, há diferença grande entre os dois achados. Pablo e et al. ${ }^{23}$ também obtiveram lacuna entre os resultados encontrados para mesmos indicadores de estado nutricional na avaliação de pacientes admitidos em hospital geral espanhol em que observaram que 63,3\% estavam desnutridos de acordo com a ASG enquanto que $88 \%$ deles apresentavam desnutrição segundo o IRN, entre eles $23 \%$ apresentaram IRN grave.

Estudos demonstram que outros métodos de triagem nutricional são capazes de mostrar que as complicações pós-operatórias de operações gastrintestinais são mais frequentes, de maior gravidade e demandam mais re-intervenções cirúrgicas para os indivíduos em risco nutricional e que o suporte nutricional estabelecido a partir desse diagnóstico de risco é capaz de reduzir as taxas de complicações ${ }^{17,26}$. Espera-se que, além das triagens, métodos menos subjetivos, como o IRN, possam colaborar em predizer presença de complicações. No presente trabalho, os pacientes que tiveram complicações apresentaram valores inferiores de IRN, sendo significativo entre a ausência de complicação e a presença de complicações não infecciosas. Entretanto, quando se pretende correlacionar com outros indicadores do estado nutricional, já no trabalho de Pablo et al. ${ }^{23}$ foi demonstrado que há correlação baixa entre ASG e IRN e corrobora com estes achados, visto que não houve correlação entre ASG e complicações, mas, a mesma foi encontrada para o IRN. No trabalho de Almeida, et al. ${ }^{1}$ com 300 pacientes cirúrgicos eletivos e não eletivos, em que $76 \%$ foram submetidos à cirurgia gastrintestinal, foi observado que o IRN não concordou com a ASG; contudo, foi sugerido que ele apresenta baixas sensibilidade e especificidade para detectar risco de desnutrição comparado com a ASG.

Algumas complicações pós-operatórias podem estar relacionadas à cicatrização da ferida operatória. De acordo com Campos et al. ${ }^{9}$, a cicatrização pós-cirúrgica depende de inúmeras variáveis e pode ser dividida em quatro fases: inflamatória, proliferação, deposição de colágeno e maturação, sendo as proteínas séricas, como a albumina, fundamentais nesse processo. Portanto, um dos fatores sistêmicos que afeta a qualidade e o tempo de cicatrização é o estado nutricional. De acordo com Oh et al. ${ }^{22}$, desnutrição segundo o IRN foi encontrada em $84,6 \%$ dos 669 pacientes no quinto dia de pós-operatório de gastrectomia por adenocarcinoma gástrico e $94 \%$ dos pacientes que apresentaram complicações na cicatrização estavam no grupo de desnutridos. Entretanto, no presente estudo, não foi analisado o tempo de cicatrização em si, mas não houve diferença do estado nutricional entre os pacientes que necessitaram e os que não precisaram de reintervenção cirúrgica durante a mesma internação hospitalar, mesmo para aqueles com valores inferiores de albuminemia, que poderiam estar relacionado à cicatrização.

Além de fundamental na cicatrização, o valor sérico de albumina é método clássico de previsão de morbidade e mortalidade, complicações pósoperatórias e tempo de hospitalização e relacionar-se com o estado nutricional, já que desnutrição leva à redução da síntese de albumina; todavia, também pode ser indicador da gravidade da doença ${ }^{15,21,29}$. Os valores do IRN são obtidos pela equação dependente do valor de albumina sérica e do percentual de perda de peso.

Lohsiriwat et al. ${ }^{19}$ verificaram que a taxa de complicações entre os pacientes com hipoalbuminemia foi maior quando comparada aos que não apresentaram albumina abaixo do adequado (37,5\% contra $21,3 \%$ ) em indivíduos submetidos procedimento cirúrgico por câncer retal, resultado semelhante no presente estudo para as complicações não infecciosas, que indicaram a presença de doenças crônicas ou de fragilidade orgânica do indivíduo desnutrido.

Quanto à mortalidade, é comum que a sobrevida seja superior para aqueles com maiores níveis de albumina e menor perda de peso ${ }^{21}$. Exemplo disso pode ser observado no estudo realizado por Gregg et al. ${ }^{15}$, em que entre todos os fatores, a albumina pré-operatória foi o que apresentou associação significativa com a mortalidade para indivíduos submetidos à cistectomia radical por câncer de bexiga. Ao contrário, outro estudo demonstrou que em relação à mortalidade, níveis de albumina não obtiveram associação estatística, assim como perda de peso, para indivíduos submetidos a tratamento cirúrgico por câncer de esôfago ${ }^{21}$. O presente estudo também não encontrou significância estatística entre mortalidade e desnutrição.

Um fato que pode ser um viés do presente trabalho é que algumas complicações pós-operatórias tiveram tempo de desenvolvimento superior ao de permanência no hospital, e esses dados não foram verificados.

Vale mencionar que a resposta imunológica, que pode ter dependência em relação ao estado nutricional e produção proteica, não teve correlação com as complicações pós-operatórias, mostrando que esse indicador não deve ser considerado como fator preditivo de complicações.

Assim, o IRN pode superestimar resultados de desnutrição, visto que a albumina sérica pode não estar relacionada exclusivamente ao estado nutricional e o percentual de perda de peso, apesar de numérico, pode não ser exato, pois utiliza dado subjetivo, que é o peso usual informado pelo paciente. Apesar dessas limitações, é método rápido e fácil e mostrou correlação com complicações não infecciosas.

Os dados do presente estudo corroboram com os achados de Schiesser et al. ${ }^{26}$, que verificaram que a incidência de risco nutricional foi maior entre pacientes com câncer (40\%) comparado com doenças benignas $(8 \%)^{5}$ e com o estudo de Pablo et al..$^{23}$ que encontraram que pacientes com câncer perdem significativamente 
mais peso comparado às outras doenças.

OIMC, um dos índices mais usados na comunidade, mais uma vez mostrou-se frágil em nível hospitalar, visto que não foram encontradas associações com as complicações pós-operatórias. Outros estudos corroboram com os achados desta pesquisa. Segundo estudo realizado por Garth et al. ${ }^{14}$, o estado nutricional avaliado por meio do IMC no momento da admissão hospitalar não apresentou correlação com nenhumas das complicações pós-operatórias analisadas por esses autores. Resultado similares foram reportados por Marin et al. ${ }^{21}$ e por Pacelli et al. ${ }^{24}$.

Estudos têm demonstrado que quanto mais desnutrido o paciente maior também o tempo de hospitalização, podendo ser até três vezes maior a probabilidade de desnutridos ficarem 15 ou mais dias hospitalizados $7,14,19,28$. Os resultados do presente estudo mostraram que os pacientes mais desnutridos complicaram mais e permaneceram maior tempo no hospital. A busca por índices apropriados de diagnóstico nutricional justifica-se para a detecção precoce da desnutrição, que se possa realizar terapia nutricional peri-operatória adequada a fim de também aumentar qualidade de vida e reduzir período de hospitalização.

\section{CONCLUSÃO}

A albumina sérica e o IRN são indicadores de estado nutricional que demonstraram melhor capacidade de predizer a ocorrência de complicações não infecciosas pós-operatórias associadas à desnutrição.

\section{REFERÊNCIAS}

1. Almeida AI, Correia M, Camilo M, Ravasco P. Nutritional risk screening in surgery: valid, feasible, easy! Clin Nutr. 2012;31(2):206-11.

2. Blackburn GL, Bistrian BR, Maini BS, Schlamm HT, Smith MF. Nutritional and metabolic assessment of the hospitalized patient. J Parenter Enteral Nutr. 1977; 1(1):11-21.

3. Blackburn GL, Thornton PA. Nutritional assessment of the hospitalized patient. Med Clin North Am. 1979; 63(5):11103-15.

4. Blackburn GL. Metabolic considerations in management of surgical patients. Surg Clin N Am. 2011; 91(3):467-80.

5. Bottoni A, Oliveira GPC, Ferrini MT, Waitzberg DL. Avaliação nutricional: exames laboratoriais. In: Waitzberg, DL. Nutrição oral, enteral e parenteral na prática clínica. 3a ed. São Paulo: Atheneu; 2000. 279-294 p.

6. Bragagnolo $R$, Caporossi FS, Dock-Nascimento DB, AguiliarNascimento JEA. Espessura do músculo adutor do polegar: um método rápido e confiável na avaliação nutricional de pacientes cirúrgicos. Rev Col Bras Cir. 2009; 36(5):371-76.

7. Burden ST, Hill J, Shaffer JL, Todd C. The British Dietetic Association. Nutritional status of preoperative colorectal cancer patients. J Hum Nutr Diet. 2010; 23(4):402-7.

8. Buzby GP, Williford WO, Peterson OL, Crosby LO, Page CP, Reinhardt $G F$, et al. A randomized clinical trial of total parenteral nutrition in malnourished surgical patients: the rationale and impact of previous clinical trials and pilot study on protocol design. Am J Clin Nutr. 1988; 47(Suppl 2):357-65.

9. Campos AC, Groth AK, Branco AB. Assessment and nutritional aspects of wound healing. Curr Opin Clin Nutr Metab Care. 2008;11: 281-8.
10. Chumlea WC, Roche AF, Steinbaugh ML. Estima 7 ting stature from knee height for persons 60 to 90 years of age. J Am Geriatr So $\neg$ C. 1985; 33(2):116-20.

11. Correia MITD, Campos ACL. Prevalence of Hospital Malnutrition in Latin America: The Multicenter ELAN Study. Nutrition. 2003; 19(10):823-5.

12. Detsky AS, McLaughlin JR, Baker JP, Johnston N, Whittaker S, Mendelson RA, Jeejeebhoy, KN. What is subjective global assessment of nutritional status? J Parenter. Enteral Nutr. 1987; 11(1):8-13.

13. Frisancho AR. New norms of upper limb fat and muscle areas for assessment of nutritional status. Am J Clin Nutr November. 1981; 34(11): 2540-5.

14. Garth AK, Newsome CM, Simmance N, Crowe TC. The British Dietetic Association Ltd. Nutritional status, nutrition practices and postoperative complications in patients with gastrointestinal cancer. J Hum Nutr Diet. 2010; 23(4): 393-401.

15. Gregg JR, Cookson MS, Phillips S, Salem S, Chang SS, Clark PE, Davis R, Stimson Jr CJ, Aghazadeh M, Smith Jr JA, Barocas AD. Effect of preoperative nutritional deficiency on mortality after radical cystectomy for bladder cancer. J Urol. 2011; 185(1):90-6.

16. Jensen GL, Mirtillo J, Compher C, Dhaliwal R, Forbes A, Figueredo Grijalba R, Ardí G, Kondrup J, Labadarios D, Nyulasi I, Castillo Pineda JC, Waitzberg D. Adult starvation and disease-related malnutrition: A proposal for etiology - based diagnosis in the clinical practice setting from the International Consensus Guidelines Comité. JPEN 2010; 34(2):156-159.

17. Jie B, Jiang ZM, Nolan MT, Efron DT, Zhu SN, Yu K, Kondrup J. Impact of nutritional support on clinical outcome in patients at nutritional risk: A multicenter, prospective cohort study in Baltimore and Beijing teaching hospitals. Nutrition. 2010; 26(11):1088-93.

18. Leandro-Merhi VA, Aquino JLB, Chagas JFS. Risco nutricional no período pré-operatório. Arq Bras Cir Dig. 2009; 22(3):143-6.

19. Lohsiriwat V, Lohsiriwat D, Boonnuch W, Chinswangwatanakul V, Akaraviputh T, Lert-akayamanee N. Pre-operative hypoalbuminemia is a major risk factor for postoperative complications following rectal cancer surgery. World J Gastroenterol. 2008; 14(8):1248-51.

20. Mahmound MK, Merril TD. Surgical Complications. In: Sabinston, Textbook of Surgery, 18a ed. New Delhi: Elsevier; 2007. cap. 15.

21. Marin FA, Lamônica-Garcia VC, Henry MACA, Burini RC. Grade of esophageal cancer and nutritional status impact on postsurgery outcomes. Arq Gastroenterol. 2010;47(4):348-53.

22. Oh AC, Kim DH, Oh SJ, Choi MG, Noh JH, Sohn TS, Bae JM, Kim S. Nutritional risk index as a predictor of postoperative wound complications after gastrectomy. World J Gastroenterol. 2012;18(7):673-8.

23. Pablo AMR, Izaga MA, Alday LA. Assessment of nutritional status on hospital admission: nutritional scores. Eur J Clin Nutr. 2003; 57:82431.

24. Pacelli F, Bossola M, Rosa F, Tortorelli AP, Papa V, Doglietto GB. Is malnutrition still a risk factor of postoperative complications in gastric cancer surgery? Clin Nutr. 2008; 27(3):398-407.

25. Ryu SW, Kim IH. Comparison of different nutritional assessments in detecting malnutrition among gastric cancer patients. World J Gastroenterol. 2010; 16(26):3310-17.

26. Schiesser M, Müller S, Kirchhoff $P$, Breitenstein S, Schäfer M, Clavien PA. Assessment of a novel screening score for nutritional risk in predicting complications in gastro-intestinal surgery. Clin Nutr. 2008; 27(4):565-70.

27. Stratton RJ, Green CJ, Elia M. Scientific criteria for defining malnutrition. In: Stratton RJ, Green CJ, Elia M. Disease-related malnutrition: an evidence based approach to treatment. Wallingford, Oxon: CABI Publishing, 2003. p. 1-34.

28. Waitzberg DL, Caiaffa WT, Correia MITD. Hospital malnutrition: the Brazilian national survey (IBRANUTRI): a study of 4000 patients. Nutrition. 2001; 17 (7):573-80.

29. Waitzberg DL, Correia MITD. Nutritional assessment in the hospitalized patient. Curr Opin Clin Nutr Metab Care. 2003; 6(5):53138.

30. World and health organization (WHO). BMI classification. [2005?] [acesso em 2012 set 19]; Disponível em: http://www.who.int/bmi/ index.jsp? introPage=intro_3.html. 